\title{
Los recursos económicos para la investigación y su impacto en estudiantes de posgrado
}

The economic resources for research and its impact on graduate students

Recursos financeiros para pesquisa e seu impacto sobre os alunos de pósgraduação

José de Jesús Peinado Camacho

Instituto Politécnico Nacional, Centro de Investigación e Innovación Tecnológica, México

jpeinadoc@ipn.mx https://orcid.org/0000-0002-2262-4565

\section{Resumen}

El objetivo del presente trabajo de investigación fue mostrar datos cuantitativos de los recursos para la investigación y su impacto en los estudiantes de posgrado del Centro de Investigación e Innovación Tecnológica del Instituto Politécnico Nacional. La pregunta que guio el presente estudio fue la siguiente: ¿cuál es la relación que existe entre los recursos para la investigación y su impacto en los estudiantes de posgrado? El diseño de la investigación fue no experimental, y la metodología que se utilizó fue cuantitativa, mientras que el enfoque del estudio fue exploratorio y descriptivo. Como técnica de investigación se utilizó el estudio de caso. Los resultados de la investigación revelaron que el número de proyectos de investigación con financiamiento interno fue disminuyendo de 14 en el año 2015 a 13 en el 2016, y 11 en el año 2017, pero en el año 2018 se incrementó a 16. Se evidenció que el monto de recursos económicos no es directamente proporcional al número de proyectos de investigación, pues si bien en el año 2018 hubo un incremento de cinco trabajos con respecto al año anterior, en esa fecha el monto de los recursos no se elevó sustancialmente. Asimismo, la cantidad de estudiantes participantes en proyectos de investigación fue decreciendo: los datos indicaron que en el 2015 participó $51.6 \%$ de los estudiantes de maestría y doctorado, 


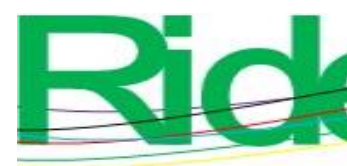

Revista Iberoamericana para la
Investigación y el Desarrollo Educativo
ISSN $2007-7467$

mensurar esses elementos nos permite descrever sua composição, conhecer sua interação e refletir sobre seus propósitos intrínsecos para otimizar sua compreensão.

Palavras-chave: ciência e administração de pesquisa, centro de pesquisa, financiamento de ciência, gastos em ciência, projeto de pesquisa.

Fecha Recepción: Agosto 2020

Fecha Aceptación: Enero 2021

\section{Introducción}

La formación de investigadores es considerada como un proceso sistemático necesario que permite concretar en un tema específico procesos relacionados con una gran cantidad de contenidos (Fonseca, 2020). Los estudiantes de posgrado aprenden a hacer ciencia al participar en grupos de investigación, ya sea en comunidades de práctica o epistémicas en las que adquirieren competencias metodológicas e intelectuales (Feldman, Divoll y Rogan-Klyve, 2013), donde pueden ver el impacto social y la importancia de su trabajo, un factor conocido para fortalecer la persistencia académica y profesional en los campos de ciencia (Weinberg, Trott y Sample, 2018).

Por eso, las preguntas de los estudiantes tienen una función importante en el aprendizaje de las ciencias y en los enfoques basados en la indagación. Para Herranen y Aksela (2019) "la enseñanza de investigación que se basa en las preguntas de los estudiantes es promisoria, siempre que no falte una visión holística de la investigación y la práctica” (p. 1). De esta forma, es factible no solo elaborar conexiones con la investigación científica de identidad, sino también enriquecer la investigación con autoconceptos de ciencia y tecnología (Rüschenpöhler y Markic, 2019). Los ejes temáticos de los estudiantes deben responder a las necesidades de campos académicos y a la resolución de problemas prácticos de ambientes laborales (Castro y López, 2020), así como del contexto social en el que están inmersos (Martínez y Márquez, 2015), vinculando la investigación con proyectos que logren impactar positivamente en el entorno (Cantú, Medina y Martínez, 2019).

Desde estas perspectivas, la formación de investigadores en su práctica científica es resultante del saber compartido por investigadores consolidados, en el camino de acceder a una comprensión de los fenómenos de la naturaleza y la sociedad (Fonseca, 2020). Al respecto, García, Pérez y Miranda (2018) mencionan que los investigadores se ven motivados a "transferir conocimiento y tecnología por la implementación del conocimiento científico 


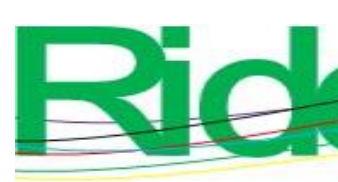

Revista Iberoamericana para la
Investigación y el Desarrollo Educativo
ISSN $2007-7467$

en situaciones reales, por la generación y el desarrollo de ideas con potencial para convertirse en innovaciones, y por la necesidad de ayudar a diversos sectores de la sociedad" (p. 52).

Sin embargo, no es realista esperar que los estudiantes alcancen el dominio total de las experiencias de investigación tradicionales a corto plazo o esperar que lleven por sí mismos una investigación científica auténtica, ya que sus experiencias deben estructurarse en grupos de investigación muy organizados con mentores que explícitamente les enseñen cómo hacer ciencia (Feldman et al., 2013). En este contexto, el papel del profesor se ve afectado por cuánto énfasis se pone en el aprendizaje de las ciencias y cuánto valor le da a las preguntas de los estudiantes (Herranen y Aksela, 2019). De este modo, los profesores realizan cambios permitiendo que los estudiantes avancen y adquieran las competencias necesarias para su formación (Peinado, 2020), lo que al mismo tiempo influye en el desarrollo profesional de los profesores, su educación formal y su participación en la investigación (Griffioen y De Jong, 2015).

Ahora bien, la forma en que se enseña a investigar guarda relación directa con cada disciplina, lo que se evidencia en los procesos de asesoría que toman diversas formas según la orientación del programa y sus lineamientos internos (Castro y López, 2020). Al respecto, Ortiz, Topete y Bustos (2018) explican:

Aún no se han logrado evitar prácticas inadecuadas en la investigación y en su formación, esto se atribuye al problema estructural de políticas, las cuales responden a intereses de poder y al diseño en la estrategia de evaluación, inapropiado para la formación de investigadores en las ciencias, lo cual es una dirección equivocada que demerita la calidad de la investigación y la academia, pues genera carga de trabajo, intereses de grupos de poder y endogamia al acaparar elementos académicos estratégicos como la dirección de tesis, aceptar estudiantes sin competencias investigativas, realizar procesos de formación simplistas o de no exigencia con jurados flexibles (p. 17).

Otro aspecto importante es que la investigación interdisciplinaria es un modo popular de producción de conocimiento que se promueve intensamente en los centros de investigación de todo el mundo (Woiwode y Froese, 2020), mediante la construcción de redes de participación organizacional en procesos y prácticas que permiten compartir valores como la confianza, el respeto, aceptar estar en desacuerdo y celebrar la diversidad (Taysum, 2016). Algunas veces, las redes de trabajo se dan por simpatía o porque ya se conocen, lo que genera un mejor trabajo, hecho por gusto y de forma natural, pues se identifican entre ellos (Ortiz, 


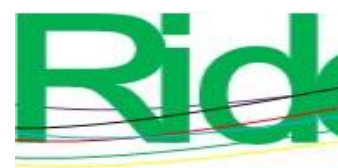

Revista Iberoamericana para la
Investigación y el Desarrollo Educativo
ISSN $2007-7467$

Topete y Bustos, 2018). No obstante, los estímulos para las propuestas multidisciplinarias no son lo suficientemente atractivos para los investigadores, pues por el número de participantes y los recursos para cada integrante son menores a los que obtendrían cada uno por separado, factor que probablemente desalienta en la creación de este tipo de proyectos (Fabila, 2014). Aun así, hay que destacar que la interdisciplinariedad puede dar respuesta a los problemas socioeconómicos y ambientales (Rosas-Baños, 2019), de ahí que sea responsabilidad de las instituciones de educación pública el promoverla e incentivarla.

\section{Recursos económicos para la investigación}

El gasto en investigación y desarrollo experimental (GIDE) es un componente del gasto nacional en ciencia, tecnología e innovación, el cual para 2017 fue de un estimado de 97166.1 millones de pesos, cifra que decreció $6.4 \%$ con respecto al año 2016. El sector que más aporto fue el público con $62.94 \%$ del total. El GIDE como porcentaje del producto interno bruto (PIB) es un indicador que otorga noción sobre los esfuerzos que realizan los países para realizar investigación y desarrollo experimental; en 2017 la relación del GIDE con el PIB en México fue 0.48 \% (Consejo Nacional de Ciencia y Tecnología [Conacyt], 2017).

México se encuentra por debajo del promedio de la relación GIDE/PIB en América Latina con 0.51, aunque por encima de Chile, Uruguay, Colombia y Panamá. Sin embargo, con países miembros de la Organización para la Cooperación y el Desarrollo Económicos (OCDE) la brecha es mayor cuando se compara con naciones como Corea del Sur (que invierte ocho veces más que México). Los países líderes en actividades científicas y tecnológicas como Alemania, Estados Unidos o Japón destinan recursos por encima del promedio OCDE, mientras que Canadá, Francia, España y Reino Unido se encuentran por debajo del promedio (aunque igualmente invierten tres y cuatro veces más que México) (Conacyt, 2017).

Por otra parte, la Organización de las Naciones Unidas para la Educación, la Ciencia y la Cultura [Unesco] (2019) público un análisis de 126 países donde se indica que solamente nueve naciones poseen más de cinco mil investigadores por cada millón de habitantes. En México solo existen 244 investigadores por cada millón de habitantes, cifra que se obtiene a partir del número de miembros del Sistema Nacional de Investigadores (SNI), si se consideran todas las áreas del conocimiento. La cifra es muy baja comparada con los países 


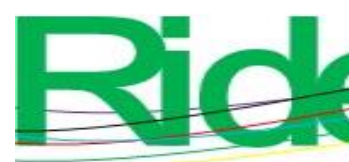

Revista Iberoamericana para la
Investigación y el Desarrollo Educativo
ISSN $2007-7467$

que cuentan con más de cinco mil investigadores por millón de habitantes, e incluso es inferior a la mayoría de los países que invierten la misma proporción de su PIB, como Chipre, Tailandia, Senegal, Macedonia y Catar, que hacen más con menos recursos y superan a México en el numero investigadores. El país con mayor número de investigadores es Noruega, con 3345, seguido por Singapur e Islandia, con 3058 y 2825, respectivamente (Román, 25 de junio de 2019). Aunado a lo anterior, en los países desarrollados la investigación científica y tecnológica se realiza principalmente en el ámbito de las unidades de investigación y desarrollo de las empresas, sin que esto impida que sus proyectos cuenten con financiamiento público (Álvarez, Chávez, Montiel, Topete y Maldonado, 2015).

Sin embargo, en países económicamente desfavorecidos la investigación parece ser una práctica desatendida particularmente (Nguyen y Van, 2018). Por eso, Álvarez et al. (2015) indican;

En los países de la región de América Latina sigue siendo necesario la participación de las instituciones de educación públicas y privadas, debido a la baja inversión tanto en la proporción del PIB como en el gasto por científico, el financiamiento casi público, mecanismos de asignación de recursos poco claros y competitivos (p. 29).

Estas concepciones institucionales están alineadas con políticas educativas neoliberales (Rosas-Baños, 2019). En este contexto de desigualdades, las instituciones de educación superior y sus centros de investigación deben encarar numerosos desafíos para la gestión eficaz de sus recursos (Mestry, 2018), ya que no cuentan con el suficiente presupuesto para el acondicionamiento de sus instalaciones (Pantoja, 2019), de ahí la necesidad de aportar más recursos a los programas de posgrado y a la investigación.

Aunado a esto, se debe trabajar en la simplificación de la burocracia administrativa (Ortiz et al., 2018), pues los trámites engorrosos desgastan a los investigadores y a los centros de investigación (Peinado, Cerecedo y Jaramillo, 2015). Por eso, se puede decir que la percepción de los profesores investigadores en cuanto a las políticas institucionales de financiamiento no es positiva, de ahí que este sea otro aspecto que se debe considerar cuando se habla de recursos para la investigación.

Además, se debe prever que en la actualidad las universidades reciben menos recursos que en épocas anteriores (Warshaw y Upton, 2019). En este sentido, Coccia y Rolfo (2013) establecen que "las debilidades organizativas y las amenazas económicas para los centros de investigación públicos son generadas por la política de investigación basada en la reducción 


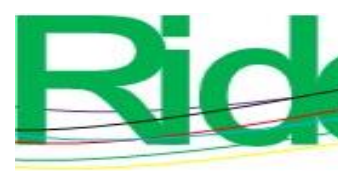

Revista Iberoamericana para la
Investigación y el Desarrollo Educativo
ISSN $2007-7467$

de los presupuestos y la reducción del personal de investigación" (p. 256). Esto repercute directamente en los recursos económicos asignados a las actividades sustantivas de instituciones como el Instituto Politécnico Nacional (IPN), por lo que podrían ser más escasos los recursos para sus centros de investigación, insumos indispensables para la docencia y la investigación (Peinado, Montoy y Torres, 2020).

Por todo lo anterior, el objetivo del presente trabajo de investigación fue mostrar datos cuantitativos de los recursos para la investigación y su impacto en los estudiantes de posgrado del Centro de Investigación e Innovación Tecnológica (CIITEC) del IPN.

La pregunta que guio el presente estudio fue la siguiente: ¿cuál es la relación que existe entre la asignación de recursos para la investigación y su impacto en los estudiantes de posgrado? Para responder esta interrogante, se indagó en el número de proyectos con financiamiento interno por año, se determinaron los montos por proyecto y por año, se establecieron los porcentajes de participación de los estudiantes en los proyectos y se cuantificó su impacto al especificar los productos de investigación que se encuentran relacionados directamente con los estudiantes de posgrado.

\section{Método}

El diseño de la investigación fue no experimental. La metodología empleada fue cuantitativa, a través de un estudio exploratorio y descriptivo. El estudio de caso ayudó a examinar y analizar en profundidad la interacción de los factores que causaron cambios en los tópicos evaluados, y como técnica de investigación permitió indagar todos los elementos que fueron importantes en la historia y el desarrollo del caso desde un enfoque complejo. De esta forma, el estudio contribuyó al conocimiento de individuos, factores y fenómenos organizacionales relacionados con el objeto de estudio.

Al inicio de la investigación se recabaron datos de los centros de investigación del IPN para respaldar, orientar y dimensionar la indagación de los tópicos relevantes para el estudio de caso, los cuales se abordaron por sus implicaciones intrínsecas, así como por los factores y elementos que lo constituyen.

En la primera etapa de la investigación relacionada con el estudio de caso se seleccionó la muestra; para ello se eligieron — del total de proyectos de investigación internos realizados por el CIITEC - aquellos con financiamiento interno correspondientes a los años 2015, 2016, 2017 y 2018, los cuales incluyen proyectos individuales, multidisciplinarios, de 


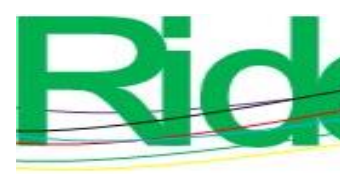

Revista Iberoamericana para la
Investigación y el Desarrollo Educativo
ISSN $2007-7467$

innovación y los del programa especial de consolidación y formación de grupos de investigación.

En la segunda etapa se recabaron los datos secundarios, los cuales debieron ser indagados y reunidos para su análisis estadístico; después fue necesario categorizarlos y finalmente explicarlos como indicadores para fundamentar la pregunta de investigación. La información fue documentada de cada uno de los departamentos del CIITEC que tienen bajo su resguardo estos datos. La tercera etapa se enfocó en obtener los datos primarios, información que fue recolectada exclusivamente para su examinación (las cifras se obtuvieron de los directores de cada proyecto de investigación). Para recopilar los datos se examinó de manera meticulosa su integración, haciendo énfasis en el tipo de proyecto y los estudiantes participantes.

\section{Resultados}

El IPN cuenta con 20 centros de investigación científica y tecnológica. El Reglamento Orgánico del IPN (2020) especifica lo siguiente:

Son unidades académicas que se encargan de realizar investigación básica, aplicada y desarrollo tecnológico, orientando sus resultados a la solución de problemas en áreas estratégicas del desarrollo nacional, para generar conocimientos que contribuyan en el avance de la ciencia y la tecnología, a la formación de recursos humanos de alto nivel, a mejorar la calidad de vida de la población, satisfacer necesidades específicas de los sectores público, social o privado y a la conservación y uso sustentable de los recursos naturales del país (p. 58).

En estos centros de investigación se encuentran adscritos 1113 docentes, 466 con doctorado, 322 con maestría, 29 con especialidad, 275 con licenciatura. De ellos, 1027 están contratados por tiempo completo y los demás se encuentran distribuidos en tres cuartos de tiempo (ocho), medio tiempo (12) y por horas (66) (IPN, 2018).

El número de investigadores SNI en los centros de investigación se ha mantenido constante con un pequeño incremento en los últimos cuatro años (de 391 en 2015 a 409 en 2016, y de 421 en 2017 a 424 en 2018). Las cantidades por nivel se presentan en la figura 1.

Figura 1. Incremento de investigadores SNI en los centros de investigación del IPN 


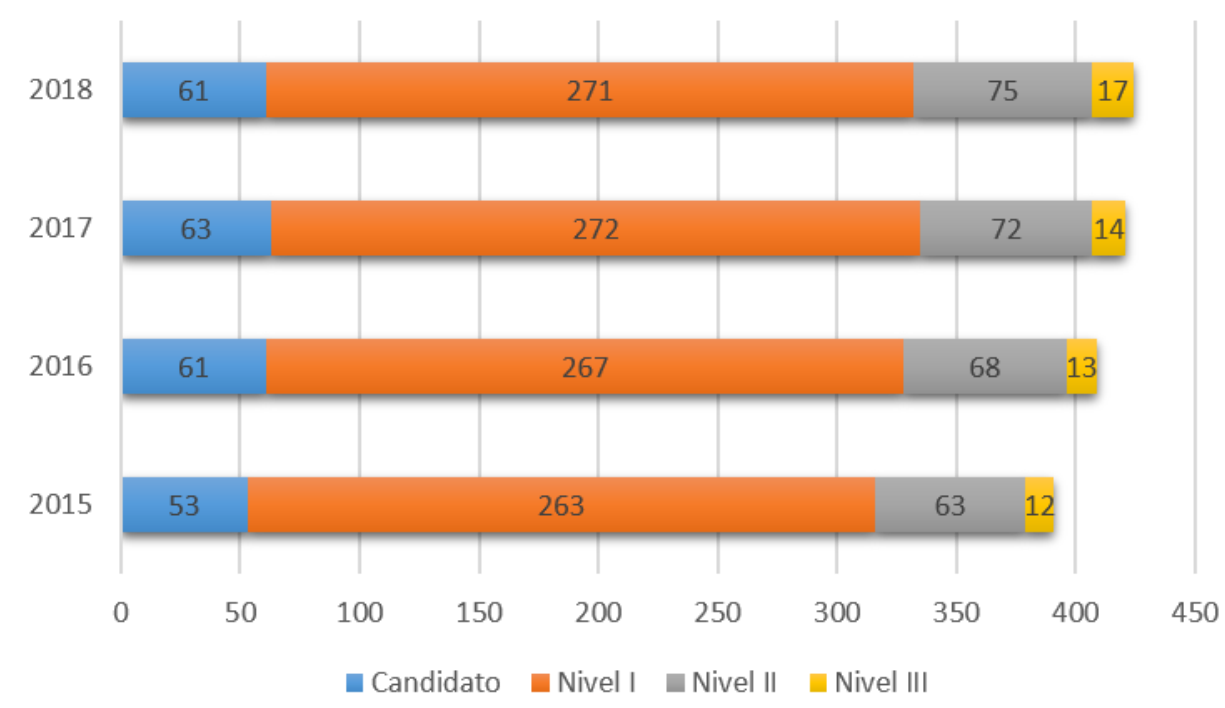

Fuente: Elaboración propia con información del IPN (2018, p. 55)

Desde el año 2015 a 2018, financió 2318 proyectos de investigación con financiamiento interno para centros de investigación (las cantidades por año se pueden observar en la figura 2).

Figura 2. Cantidad de proyectos en centros de investigación del IPN

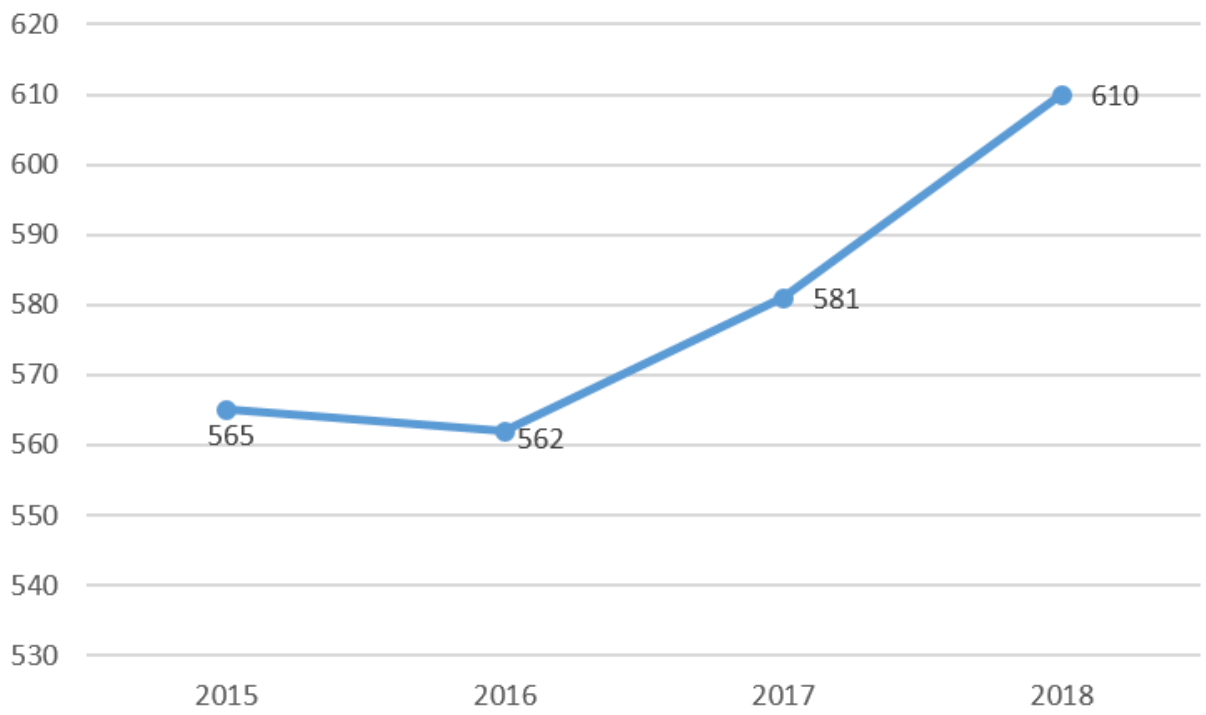

Fuente: Elaboración propia con información del IPN (2018, p. 53) 

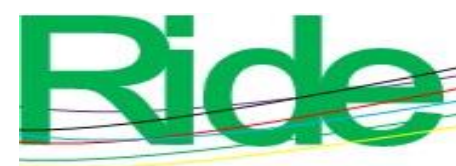

Revista Iberoamericana para la Investigación y el Desarrollo Educativo ISSN $2007-7467$

El acumulado total de recursos económicos de proyectos con financiamiento interno para los centros de investigación fue de 195232822.07 millones de pesos. Los montos por cada año se pueden ver en la figura 3.

Figura 3. Montos de los proyectos en centros de investigación del IPN

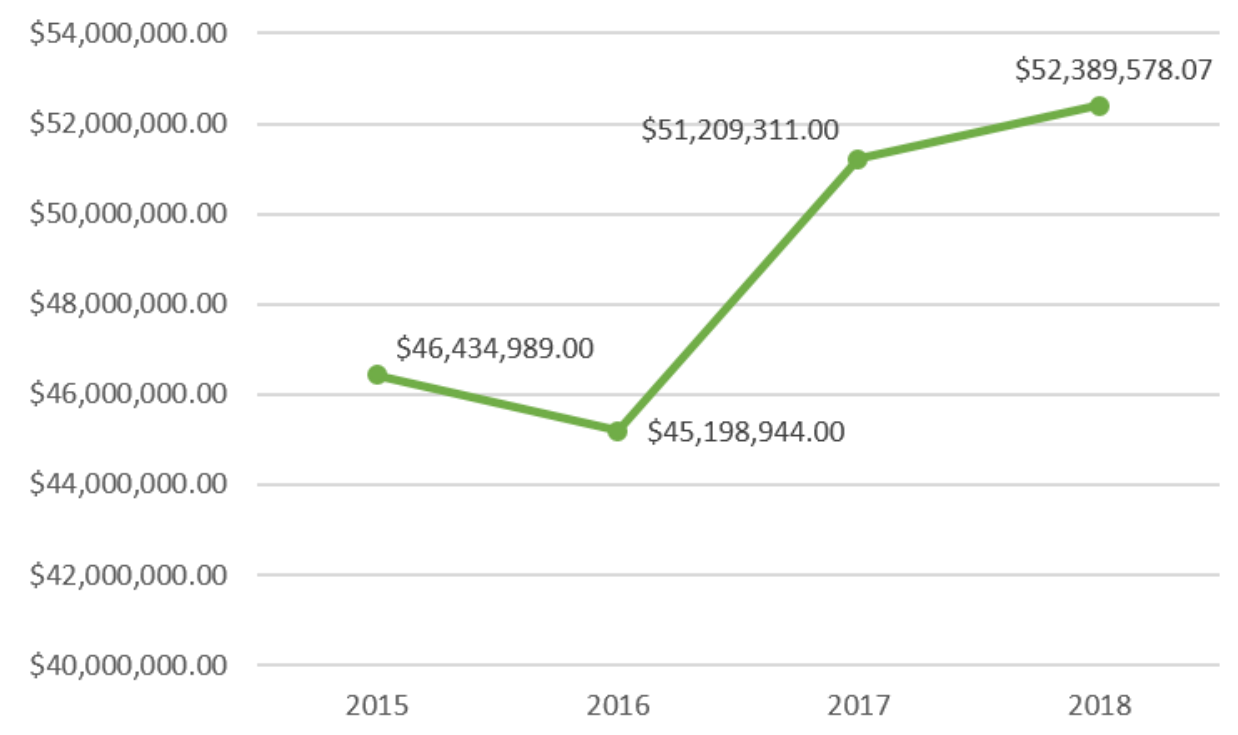

Fuente: Elaboración propia con información del IPN (2018, p. 54)

El total general de académicos en estos proyectos fue de 5943, mientras que el total de alumnos fue de 5051. En la figura 4 de pueden ver las cantidades de participantes en los últimos cuatro años.

Figura 4. Participantes en proyectos de centros de investigación del IPN

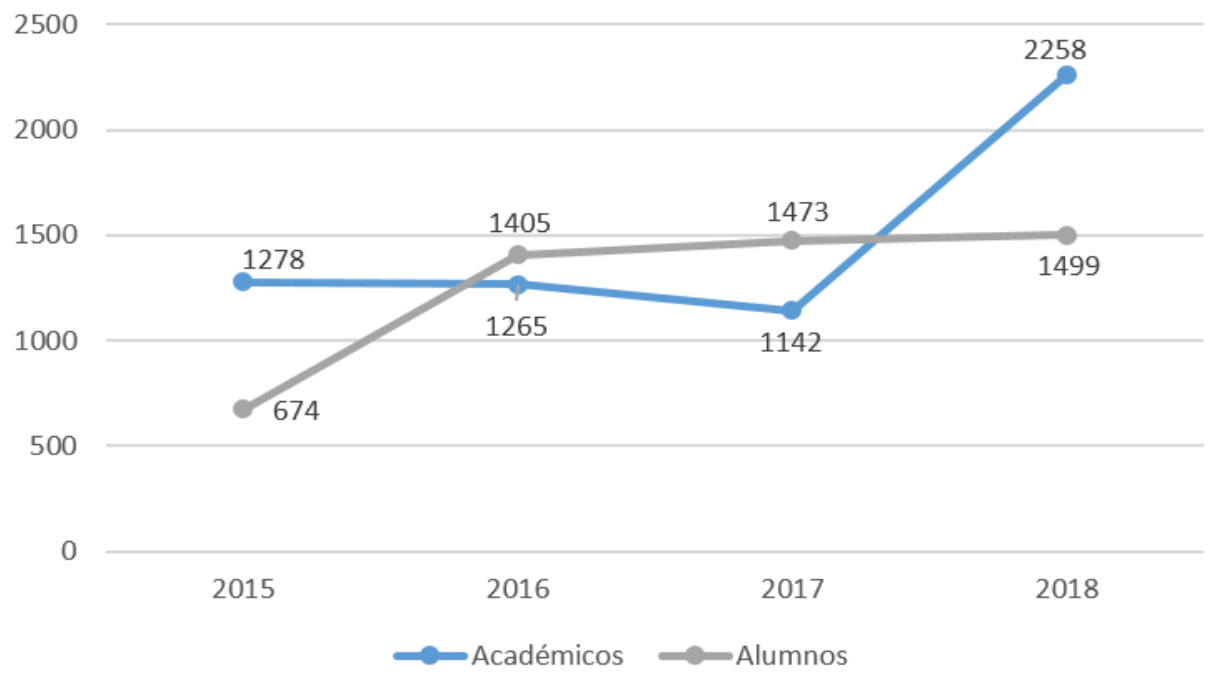

Fuente: Elaboración propia con información del IPN (2018, p. 54) 


\section{Estudio de caso del Centro de Investigación e Innovación Tecnológica}

El CIITEC fue fundado con el objetivo de contribuir al fortalecimiento de los sectores productivos y educativos a través de la investigación aplicada, el desarrollo tecnológico y la innovación tecnológica (Bonilla y Peinado, 2014). Su misión se enfocó en formar recursos humanos de alta capacidad creativa e innovadora para integrarse a los sectores productivo y de servicios, con programas académicos de posgrado pertinentes, asesorías, servicios tecnológicos y mediante la creación y ejecución de proyectos vinculados y de investigación (Peinado, Cerecedo y Jaramillo, 2016).

El programa de maestría en Tecnología Avanzada del CIITEC fue creada en el año 2007 con el objetivo de formar recursos humanos de alto nivel académico a través de la investigación, el desarrollo tecnológico y la innovación. La estructura curricular de la maestría fue planeada para cuatro semestres, con unidades de aprendizaje de especialización, complementarias, de innovación y tres seminarios de investigación (Bonilla y Peinado, 2014).

Los aspirantes deben tener interés para desarrollar investigaciones, aplicando el método científico que conduzca a la innovación. Por ello, se requieren habilidades de análisis, síntesis y de razonamiento abstracto para desarrollar actividades experimentales. Asimismo, deben dominar el idioma inglés u otra lengua extranjera (Peinado y Jaramillo, 2018).

El programa de doctorado en Tecnología Avanzada del CIITEC contempla tres seminarios departamentales obligatorios; la materia de innovación se incluye solo en caso de que el estudiante la requiera y las materias optativas se incorporan si el alumno necesita desarrollar fortalezas adicionales para realizar su investigación (Bonilla y Peinado, 2014). Los aspirantes a ingresar al doctorado deben tener interés para desarrollar investigación que conduzca al fortalecimiento de la ingeniería, la tecnología y la innovación, tomando en cuenta los proyectos afines a los desarrollados por los integrantes del colegio de profesores. Debe ser egresado de alguna maestría en tecnología, ingeniería o áreas afines, y tener interés en desarrollar investigación original básica y/o aplicada, innovación tecnológica y capacidad académica en el área de ingeniería y tecnología avanzadas. Por ello, se requieren habilidades de análisis y síntesis de razonamiento abstracto para desarrollar actividades experimentales, que hayan elaborado su tesis de maestría preferentemente por investigación o desarrollo de prototipos. Debe dominar el idioma ingles u otra lengua extranjera (Peinado y Jaramillo, 2018). 

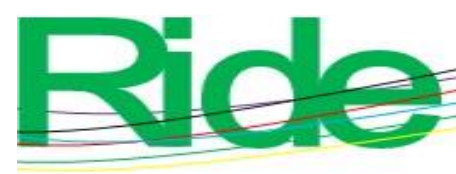

Revista Iberoamericana para la Investigación y el Desarrollo Educativo ISSN $2007-7467$

El núcleo académico básico del Colegio de Profesores del CIITEC está integrado por 12 docentes, cuatro mujeres $(33.33 \%)$ y nueve hombres $(66.67 \%)$. Todos tienen doctorado, tres lo realizaron en México y nueve en el extranjero (75\%) (seis en Francia, uno en Estados Unidos, uno en Inglaterra y uno en Japón). De ellos, 11 pertenecen al SNI, 5 son nivel dos $(41.67 \%)$ y 6 son nivel uno $(50.00 \%)$. Once tienen nombramiento de profesor colegiado y uno de asignatura. Referente al núcleo académico ampliado, está formado por siete personas: dos mujeres $(28.57 \%)$ y cinco hombres $(71.42 \%)$. Dos tienen grado de doctor $(28.57 \%)$ y cinco de maestro en ciencias $(71.42 \%)$. Todos tienen nombramiento de profesor de asignatura, ninguno pertenece al SNI y todos realizaron sus estudios de posgrado en México (Peinado, Mayagoitia y Cruz, 2019).

El primer paso para desarrollar el estudio de caso consistió en determinar el número de proyectos por año en el CIITEC. La figura 5 especifica estas cantidades en los años 2015, 2016, 2017 y 2018. Cabe resaltar que en los proyectos individuales se consideraron los proyectos de investigación del programa especial de consolidación y formación de grupos de investigación, estos solamente son dos en el año 2018.

Figura 5. Cantidad de proyectos con financiamiento interno por año

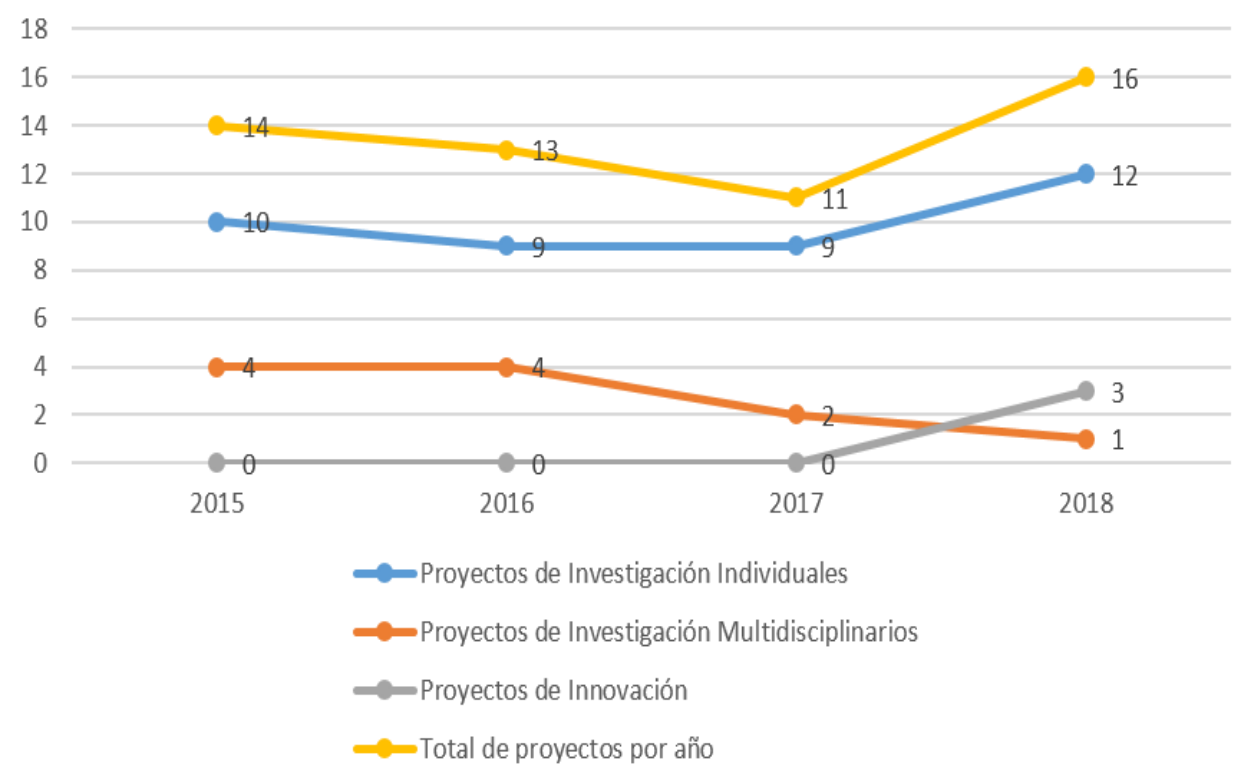

Fuente: Elaboración propia

Entre los resultados se encontraron algunas variaciones. La figura 5 permite ver que el número de proyectos de investigación con financiamiento interno disminuyó del año 2015 al año 2017, pero en el año 2018 se incrementó. Cabe resaltar que los proyectos 

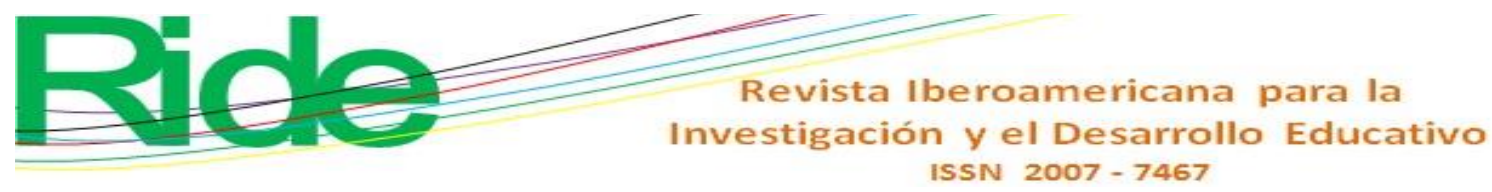

multidisciplinarios disminuyeron a uno en el último año, los proyectos individuales se incrementaron y se incursionó en los proyectos de innovación.

Otro punto importante de mencionar es que apenas en el año 2018 se incursionó en proyectos de innovación. Esto se podría justificar porque sus investigadores son principalmente SNI, de ahí que sus productos de investigación sean diferentes a los que surgen de un proyecto de innovación. Conviene decir que los profesores que hasta ahora han obtenido este tipo de proyectos en el CIITEC son ingenieros con grado de maestría, dos mujeres y un hombre, no tienen nombramiento del SNI y carecen de estímulos económicos adicionales.

El segundo paso fue indagar los montos de los proyectos por año. En la figura 6 se pueden observar las cantidades totales de los proyectos con financiamiento interno de los años 2015 al 2018. La figura compila los proyectos individuales, multidisciplinarios y de innovación.

Figura 6. Montos de proyectos con financiamiento interno de 2015 a 2018

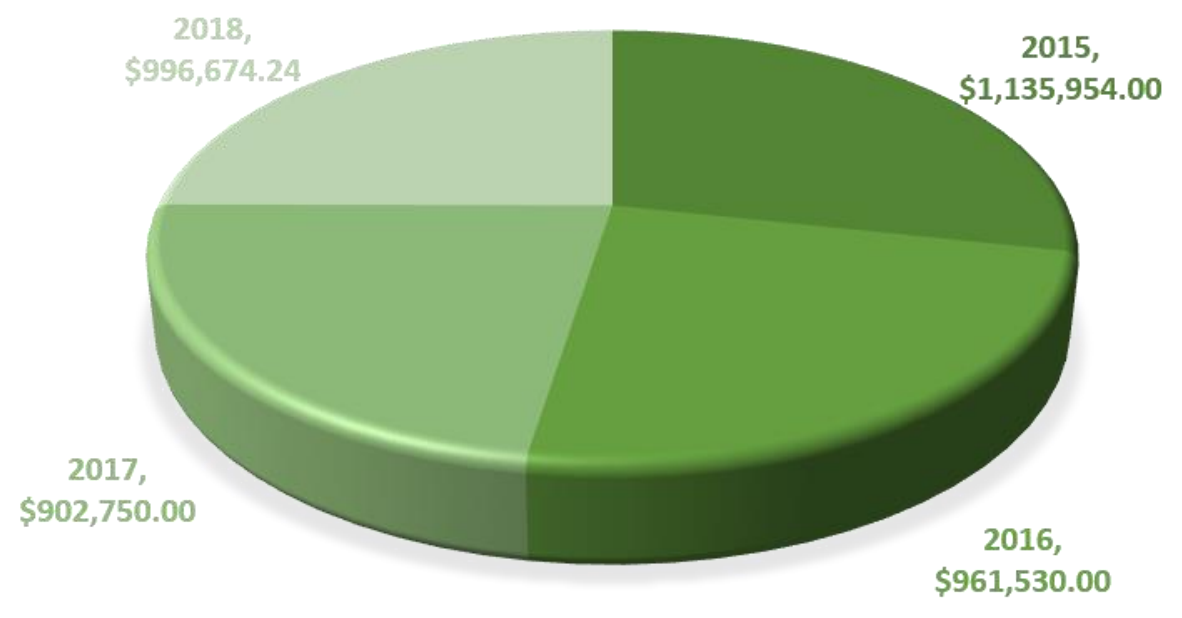

Fuente: Elaboración propia

Se evidenció que el monto de recursos económicos no es directamente proporcional al número de proyectos de investigación con financiamiento interno por año. Lo anterior se observó en la figura 5, pues la cantidad de proyectos se incrementó en el año 2018 en cinco con respecto al año anterior; también aumentó la cantidad de recursos, pero no de la misma forma, pues en el año 2015 el número de proyectos fue menor que en el año 2018, pero la cantidad de recursos asignados fue mayor. 

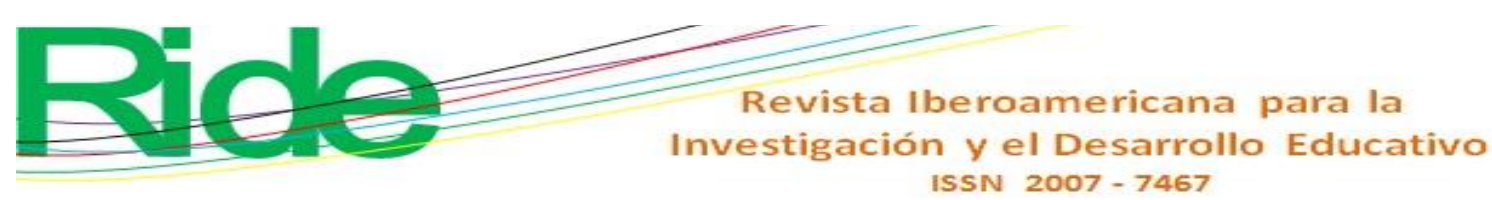

En el tercer paso se determinó el impacto de los proyectos con financiamiento interno en los estudiantes de posgrado del CIITEC, por lo que se realizó el siguiente procedimiento: primero se solicitó al Departamento de Recursos Humanos y Financieros el número de proyectos autorizados por la SIP, los montos de cada proyecto, así como el director de cada uno de los proyectos del año 2015 al año 2018. Después se gestionó en el Departamento de Posgrado del CIITEC un listado de los estudiantes inscritos en los programas de maestría y doctorado, con fecha de ingreso, de egreso y profesor director de tesis, del año 2011 al año 2018. Posteriormente se indagó con los profesores-investigadores del CIITEC cuántos y qué estudiantes habían participado en sus proyectos de investigación SIP del año 2015 al año 2018. Luego, con los datos proporcionados por los profesores sobre los estudiantes participantes, se verificó con las listas de estudiantes inscritos en los programas de maestría y doctorado, que proporcionó el Departamento de Posgrado del CIITEC. El número de proyectos y el director de cada proyecto se verificaron con la información que proporcionó el Departamento de Recursos Humanos y Financieros, lo que dio certeza sobre el profesor investigador y los estudiantes que participaron en sus proyectos. La figura 7 muestra los datos encontrados.

Figura 7. Participación de estudiantes en proyectos con financiamiento interno

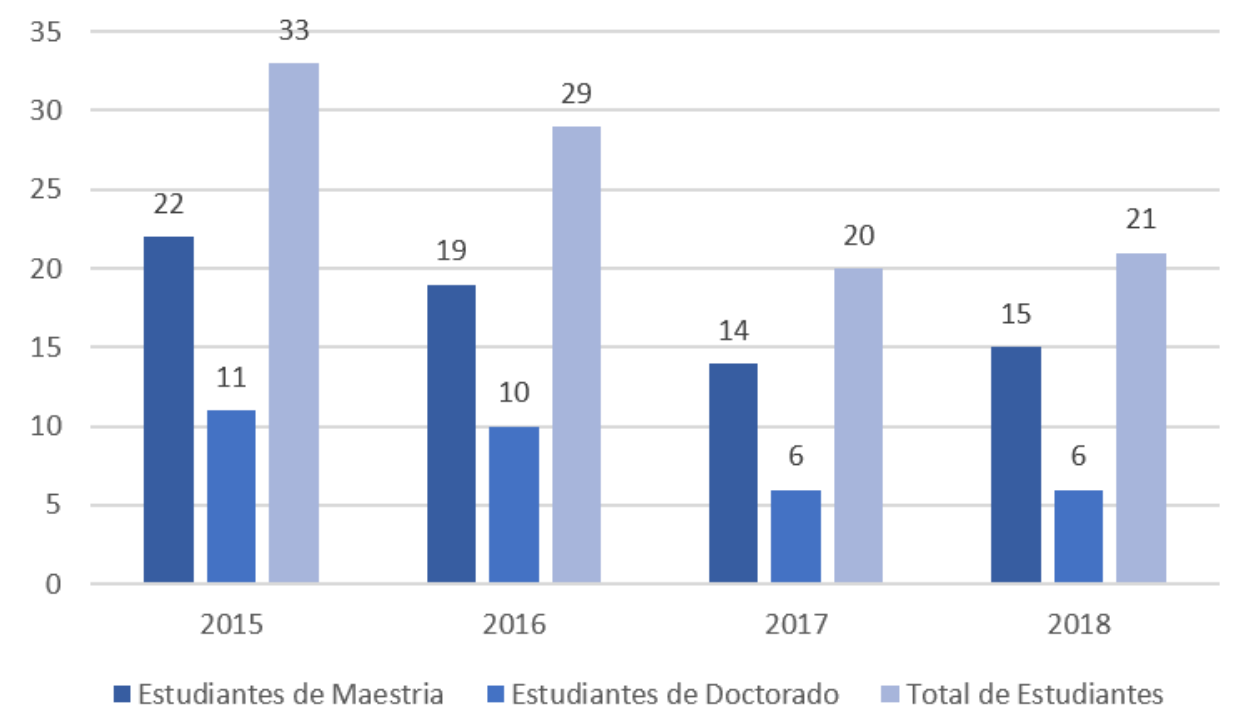

Fuente: Elaboración propia

En la figura 7 se observa que la cantidad de estudiantes participantes en proyectos de investigación ha venido decreciendo, así como el número de proyectos del año 2015 al 2017. Esta tendencia no se puede considerar como un factor, pues no es proporcional. Esto se debe 

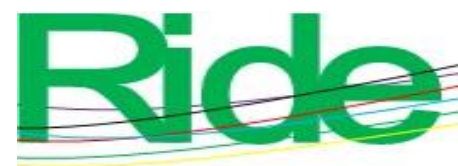

Revista Iberoamericana para la Investigación y el Desarrollo Educativo ISSN 2007 - 7467

Para mostrar las estadísticas de estos productos de investigación se tomó como referente la categorización que actualmente tiene el CIITEC sobre su línea innovadora de investigación aplicada y desarrollo tecnológico, que es multidisciplinaria y fue definida como ingeniería y tecnología avanzada. Su objetivo es desarrollar, adaptar, innovar y aplicar conocimientos en el campo de la ingeniería y la tecnología (Peinado, Mayagoitia y Cruz, 2019). A continuación, se presenta la figura 9, donde se observan las publicaciones realizadas en los últimos tres años por disciplina de investigación.

Figura 9. Artículos de investigación por disciplina de investigación

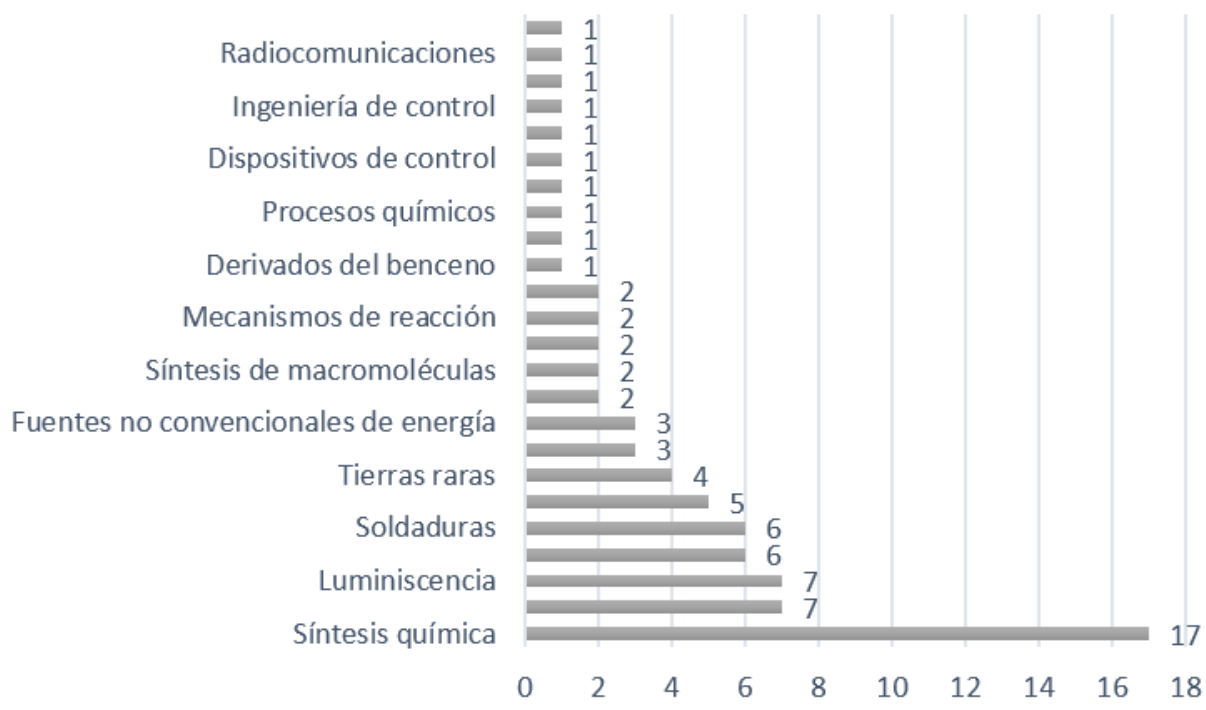

Fuente: Elaboración propia con información del Departamento de Posgrado del CIITEC

La participación de los tesistas en los proyectos de investigación contribuye en múltiples sentidos, por ejemplo, el aprendizaje, la formación en el campo de la investigación y la contribución que hacen a los profesores en su línea de generación y aplicación del conocimiento. Con respecto a las tesis de maestría, la figura 10 exhibe 116 trabajos por disciplina de investigación. 

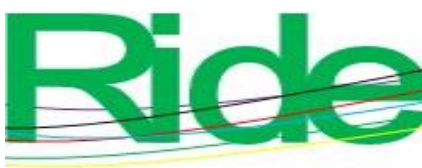

Revista Iberoamericana para la Investigación y el Desarrollo Educativo ISSN 2007 - 7467

Figura 10. Tesis de maestría por disciplina de investigación

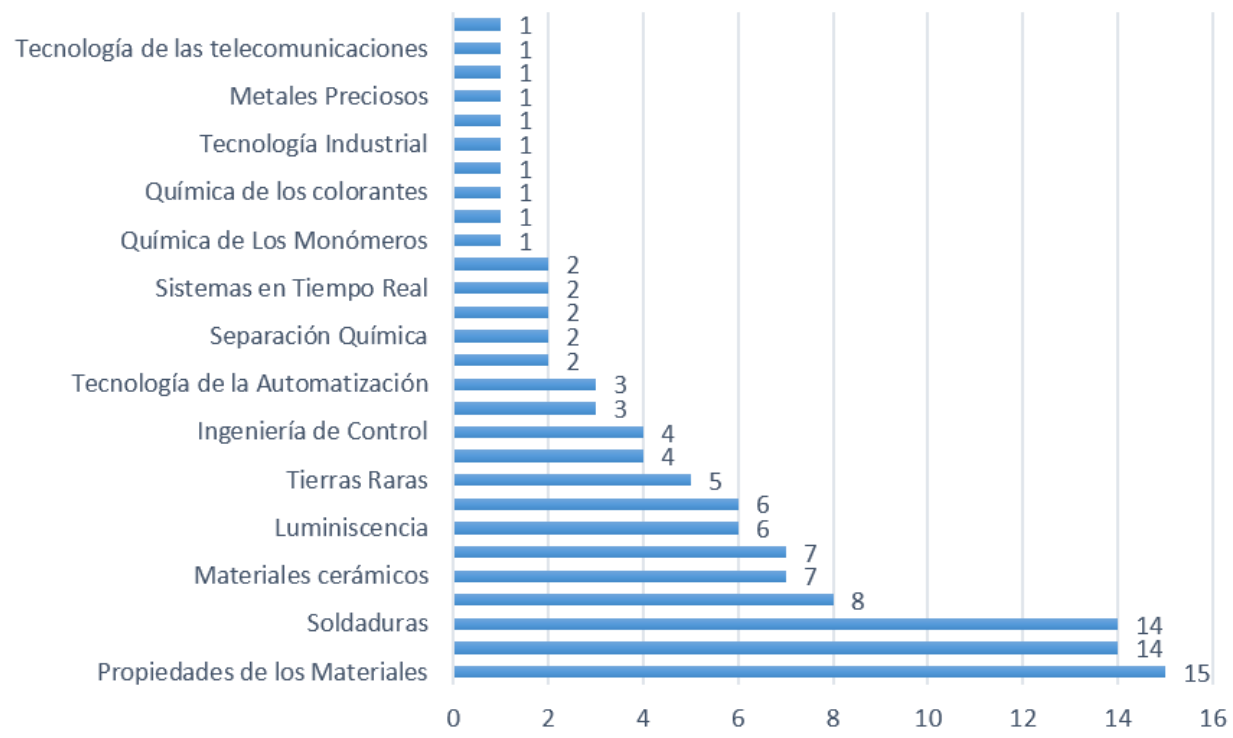

Fuente: Elaboración propia con información del Departamento de Posgrado del CIITEC

Acerca de los trabajos de tesis de los estudiantes de doctorado, en la figura 11 se presentan 39 trabajos también por disciplina de investigación.

Figura 11. Tesis de doctorado por disciplina de investigación

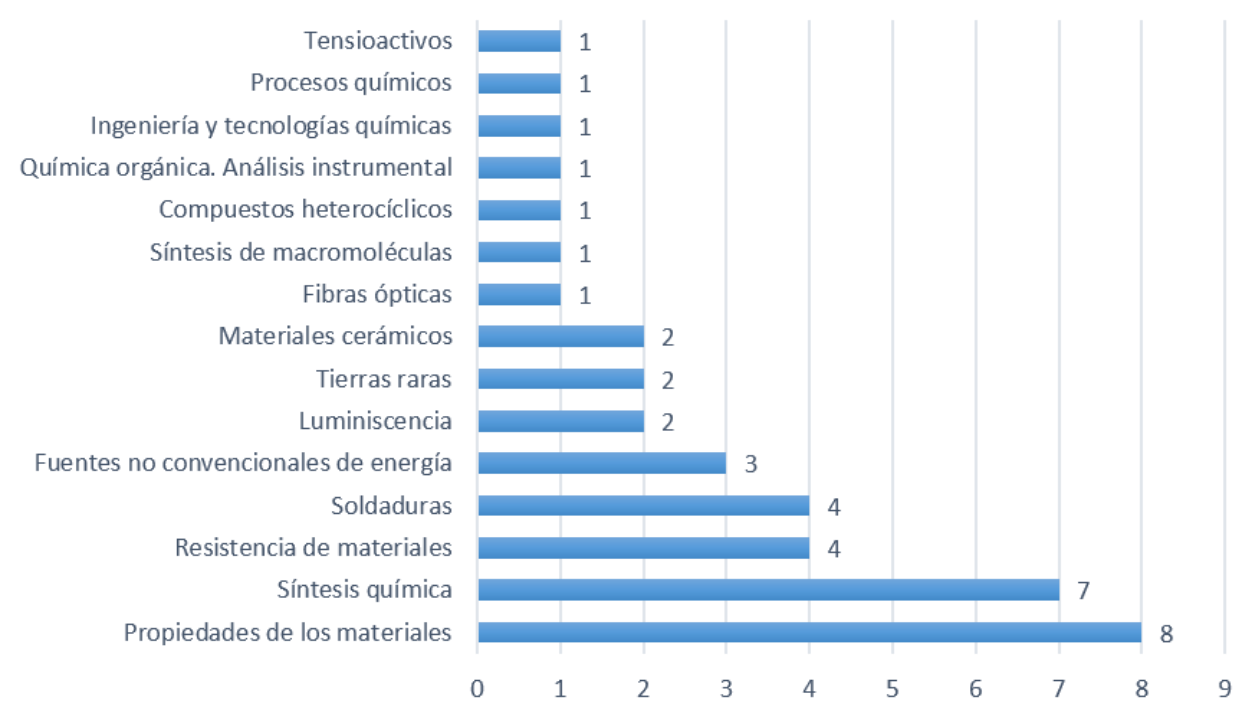

Fuente: Elaboración propia con información del Departamento de Posgrado del CIITEC 


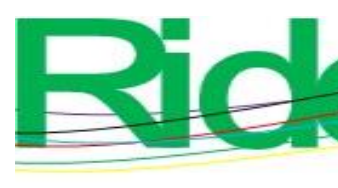

Revista Iberoamericana para la Investigación y el Desarrollo Educativo ISSN 2007-7467

\section{Discusión de resultados}

La investigación como la docencia generalmente son consumidoras de recursos económicos, de ahí que las IES deban destinar gran cantidad de ellos para realizar estas dos funciones sustantivas. Pero los recursos no son inconmensurables, y su dispersión tiene que ser adecuada a las necesidades y justificada en la factibilidad de cada investigación. De aquí que su medición sea factible, trascendente y necesaria para observar su valiosa contribución.

La orientación a la investigación se centra en la formación de investigadores y en la generación de conocimientos (Castro y López, 2020), por lo que el desarrollo de capacidades de autogestión en el proceso de investigación gira en torno a la aptitud para investigar, cualidades, habilidades y labor asignada (Fonseca, 2020). En este sentido, los estudiantes que enfrentan distintas problemáticas con frecuencia plantean propuestas para resolverlas, lo que demuestra su nivel de creatividad y responsabilidad (Peinado, 2020). Por eso, su participación en los proyectos de investigación contribuye a la solución de distintas incógnitas desde un enfoque fresco e innovador, y no solo con aportación de trabajo técnico.

Considerar la formación de los jóvenes como aprendices científicos es equiparable a la transmisión de un oficio, lo que requiere acompañamiento, instrucción, y orientación (Fonseca, 2020). Por ello, los estudiantes que ingresan a un grupo de investigación salen como investigadores novatos, técnicos competentes o productores de conocimiento (Feldman et al., 2013).

Según esta percepción, y a partir de los resultados de esta investigación, resulta necesario incorporar a más estudiantes en los proyectos, ya que su participación varía aproximadamente en $50 \%$ del total de los estudiantes en maestría y en doctorado. Una mayor inclusión brindaría más trabajo de campo y favorecería la cercanía con los profesores investigadores que guían el trabajo.

Por otra parte, la tendencia a realizar investigaciones interdisciplinarias está influenciada por factores estructurales como los programas de estudio y sus académicos (Martínez y Márquez, 2015; Woiwode y Froese, 2020). Este tipo de labores conjuntas permite acceder al conocimiento de otras disciplinas e incrementa el trabajo organizado y vinculante (García et al., 2018), lo que ofrece mejores resultados porque se atiende un problema desde diferentes enfoques (Fabila, 2014).

Ahora bien, en el caso del CIITEC, se puede decir que el trabajo interdisciplinario en los proyectos de investigación es un elemento que se fomenta y prevalece, lo que se evidencia 

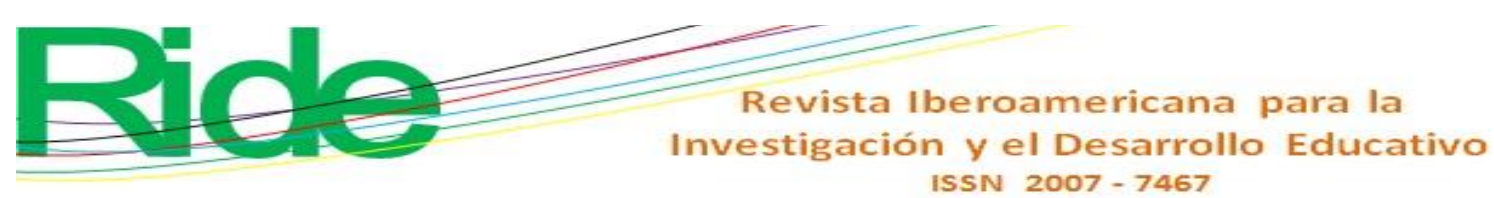

en los artículos de investigación y las tesis de maestría y de doctorado. Asimismo, vale señalar que si bien es una necesidad primordial la aportación de más recursos para la investigación y los programas de posgrado (Ortiz et al., 2018), los resultados del presente trabajo demuestran que no siempre los proyectos aumentan con más capital, el cual se encuentra a discreción de las autoridades que dispersan los recursos.

Aun así, cabe resaltar que los centros de investigación del IPN como el CIITEC no pueden subsistir únicamente con el presupuesto federal otorgado o con los recursos destinados a los proyectos (Peinado et al., 2020), por lo que se deben emprender otras acciones que permitan obtener mayor liquidez para realizar las actividades de investigación (Peinado et al., 2015).

En respuesta a la problemática de más recursos económicos para la investigación y a manera de propuesta-, la vinculación representa un medio importante para la obtención de recursos como función de apoyo; asimismo, el proceso administrativo también los concentraría y distribuiría. Estos esfuerzos incidirían positivamente en el posgrado desde dos perspectivas: la primera en el ámbito económico y la segunda en los estudiantes de posgrado, quienes se acercarían al sector industrial o de servicios para obtener experiencia y para aplicar sus conocimientos con responsabilidad social y a favor de la sustentabilidad ambiental. Para ilustrar el punto anterior la figura 12 presenta estas funciones.

Figura 12. Funciones sustantivas y de apoyo en el CIITEC

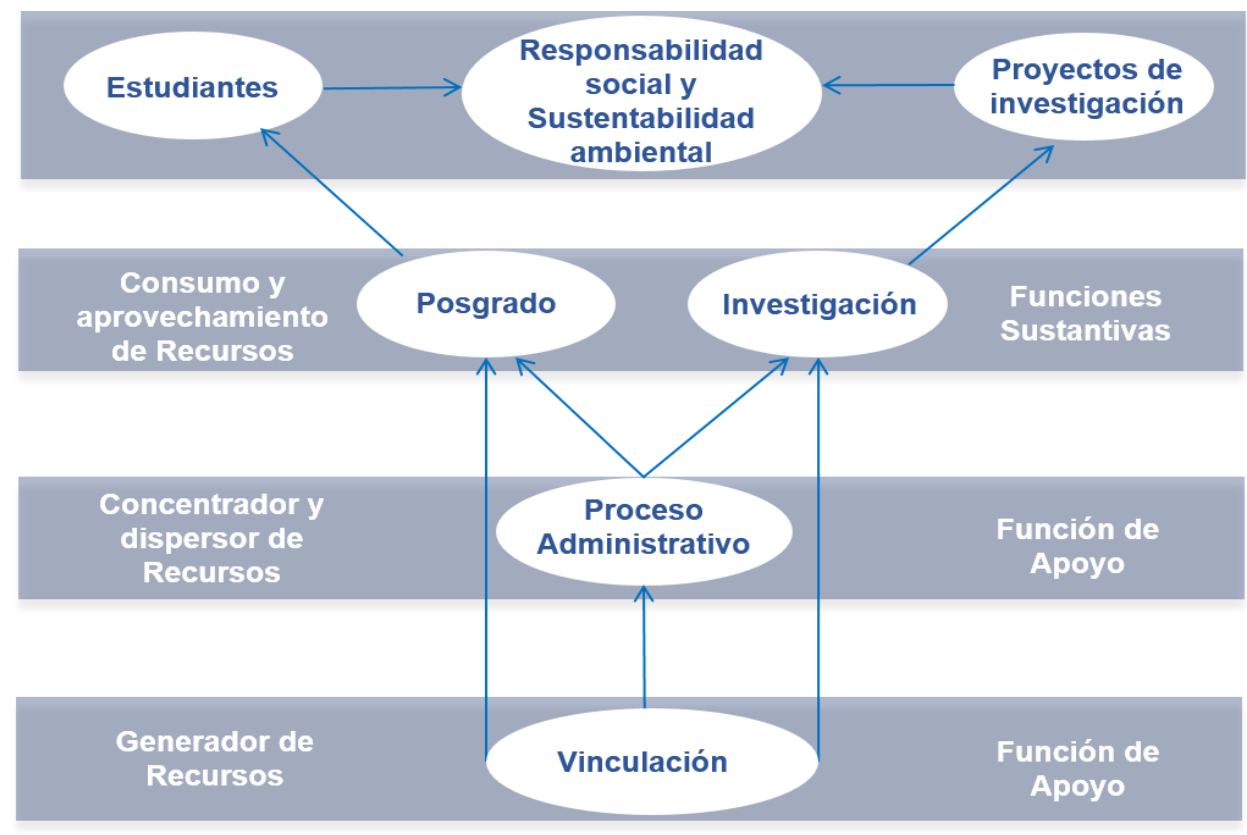

Fuente: Elaboración propia 


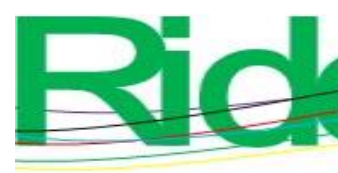

Revista Iberoamericana para la
Investigación y el Desarrollo Educativo
ISSN $2007-7467$

Finalmente, se puede decir que la importancia de esta investigación se fundamentó en confirmar y ampliar el conocimiento sobre el tópico estudiado, lo que justificó la parte crítica del caso. La perspectiva de unicidad en la investigación se manifestó al ser irrepetible y característico por los sujetos que intervienen en el contexto y por las circunstancias que lo hacen aún más diferente (Peinado et al., 2020). Adicionalmente, el estudio permitió ver su carácter revelador al observar y analizar el fenómeno, pues se evidenció la situación concreta de la asignación de recursos para la investigación y su impacto en los estudiantes de posgrado. Asimismo, permitió conocer la descripción detallada y exhaustiva de la situación real que actualmente impera, y ofreció información pormenorizada y completa de las circunstancias, fenómenos, aspectos y elementos que lo integran. Por lo anterior, es positivo desatacar que el estudio encontró las evidencias cuantitativas indagadas. Entre las limitaciones, sin embargo, se puede señalar que no es generalizable o concluyente.

\section{Conclusiones}

Producir ciencia es una tarea sublime, ya que es la base del desarrollo de un país. La investigación es una tarea importante y fundamental porque a través de su desarrollo se han encontrado soluciones para mejorar la calidad de vida de los seres humanos, aunque para ello se requieren recursos. En tal sentido, la asignación de los recursos para la investigación está directamente relacionada al posgrado con base en dos antecedentes. El primero se refiere a los recursos económicos necesarios para realizar la investigación, a través de la compra de diversos materiales y la contratación de distintos servicios. El segundo apunta a la participación de los estudiantes en los proyectos de investigación, lo que contribuye a fomentar su propio aprendizaje, así como las líneas de generación y aplicación del conocimiento.

Los proyectos de investigación, por tanto, son un elemento inherente a la existencia de los posgrados en el IPN, en especial los que se encuentran en el PNPC de Conacyt. Los subproductos que se derivan de ellos como las tesis y las publicaciones son evidencia tangible de su impacto, así como los factores cualitativos que proveen, como la participación de estudiantes del IPN, la formación de nuevos investigadores, además del crecimiento, desarrollo y consolidación de los ya formados.

Dentro de las actividades sustantivas de un centro de investigación como el CIITEC están la docencia y la investigación, ambas relacionadas a los profesores y a los estudiantes. 

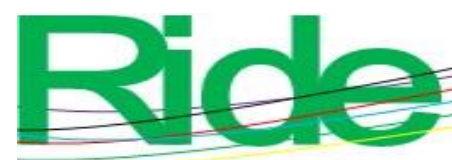

Revista Iberoamericana para la Investigación y el Desarrollo Educativo

ISSN $2007-7467$

Estos cuatro elementos deben generar una simbiosis que gestione el vínculo de estos componentes, los cuales se benefician en una correlación recíproca. Estudiar y medir los elementos mencionados, permite describir su composición, conocer su interacción y atender sus propósitos intrínsecos para conseguir la conjunción ideal y mejorar su entendimiento.

\section{Futuras Líneas de Investigación}

Es pertinente continuar investigando este tema para reforzar esta línea de investigación. Para futuros trabajos se recomienda ampliar el estudio a otros centros de investigación del IPN, de forma separada o simultánea. De igual modo, se sugiere aumentar la dimensión de este tópico, incluyendo el presupuesto federal, los recursos autogenerados, los estímulos económicos (becas) que reciben los estudiantes y los profesores investigadores. También se debe considerar la implicación de la sustentabilidad ambiental y el impacto social que tienen los proyectos de investigación y su influencia en los estudiantes de posgrado.

\section{Agradecimientos}

El autor agradece al Instituto Politécnico Nacional las facilidades otorgadas para la realización de esta investigación, a partir de la autorización del proyecto de investigación SIP20190167, titulado La asignación de recursos para la investigación y su impacto en el posgrado. 


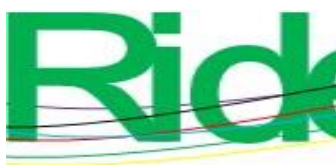

Revista Iberoamericana para la Investigación y el Desarrollo Educativo ISSN $2007-7467$

\section{Referencias}

Álvarez, I., Chávez, F., Montiel, J., Topete, C. y Maldonado, N. (2015). Aventuras científicas y tecnológicas de académicos mexicanos. Experiencia de cuatro décadas 1960-2000. México: LIMUSA.

Bonilla, A. y Peinado, J. (2014). Semblanza del Centro de Investigación e Innovación Tecnológica del IPN. Hechos que le dieron origen y su evolución. México: Taller Abierto.

Cantú, I., Medina, A. y Martínez, F. (2019). Semillero de investigación: estrategia educativa para promover la innovación tecnológica. RIDE Revista Iberoamericana para la Investigación y el Desarrollo Educativo, 10(19), 1-25. Doi: https://doi.org/10.23913/ride.v10i19.505

Castro, S. y López, E. (2020). Aproximación a la orientación de las maestrías a través de la voz de sus agentes. IE Revista de Investigación Educativa de la REDIECH, 11(733), 1-21. Doi: http://dx.doi.org/10.33010/ie_rie_rediech.v11i0.733.

Coccia, M. and Rolfo, S. (2013). Human resource management and organizational behavior of public research institutions. International Journal of Public Administration, 36(4), 256-268. Doi: https://doi.org/10.1080/01900692.2012.756889

Consejo Nacional de Ciencia y Tecnología [Conacyt] (2017). Informe general del estado de la ciencia, la tecnología y la innovación 2017. Recuperado de http://www.siicyt.gob.mx/index.php/transparencia/informes-conacyt/informegeneral-del-estado-de-la-ciencia-tecnologia-e-innovacion/informe-general2017/4813-informe-general-2017/file

Fabila, L. (2014). Diez años de apoyo a la investigación científica básica por el CONACYT. Perfiles Latinoamericanos, 1(43), 55-76. Recuperado de https://perfilesla.flacso.edu.mx/index.php/perfilesla/article/view/36

Feldman, A., Divoll, K. and Rogan-Klyve, A. (2013). Becoming researchers: The participation of undergraduate and graduate students in scientific research groups. Science Education, 97(2), 218-243. Doi: https://doi.org/10.1002/sce.21051

Fonseca, J. (2020). La formación de investigadores: oficio y conocimiento pertinente ante los avances de la ciencia y la tecnología. RIDE Revista Iberoamericana para la Investigación y el Desarrollo Educativo, 10(20), 32-50. Doi: https://doi.org/10.23913/ride.v10i20.621 


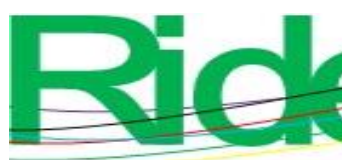

Revista Iberoamericana para la
Investigación y el Desarrollo Educativo
ISSN $2007-7467$

García, O., Pérez, R. y Miranda, A. (2018). Los profesores-investigadores universitarios y sus motivaciones para transferir conocimiento. Revista Electrónica de Investigación Educativa, 20(3), 43-55. Doi: https://doi.org/10.24320/redie.2018.20.3.1754

Griffioen, D. and De Jong, U. (2015). Implementing research in professional higher education: Factors that influence lecturers' perceptions. Educational Management Administration \& Leadership, 43(4), 626-645. Doi: https://doi.org/10.1177/1741143214523008

Herranen, J. and Aksela, M. (2019). Student-question-based inquiry in science education. Studies in Science Education, 55(1), 1-36. Doi: https://doi.org/10.1080/03057267.2019.1658059

Instituto Politécnico Nacional [IPN] (2017). Reglamento de Estudios de Posgrado del Instituto Politécnico Nacional. Gaceta Politécnica, 18(54), 34-58. Recuperado de https://www.ipn.mx/assets/files/normatividad/docs/reglamentos/GAC-EXT1358.pdf

Instituto Politécnico Nacional [IPN]. (2018). Informe anual de actividades 2018. México: IPN. Recuperado de https://www.ipn.mx/informeanual2018.pdf

Instituto Politécnico Nacional [IPN] (2020). Reglamento de Orgánico del Instituto Politécnico Nacional. Gaceta Politécnica, 18(56), 3-65. Recuperado de https://www.ipn.mx/assets/files/ccs/docs/gaceta-extraordinaria/2020/03/reglamento1541.pdf

Martínez, F. y Márquez, A. (2015). Los programas de posgrado. En Bonilla, M. (ed.), Diagnóstico del posgrado en México: Nacional (pp. 39-79). México D. F., México: COMEPO. Recuperado de https://www.posgrado.unam.mx/sitios_interes/documentos/comepo_regiones.pdf

Mestry, R. (2018). The role of governing bodies in the management of financial resources in South African no-fee public schools. Educational Management Administration \& Leadership, 46(3), 385-400. Doi: https://doi.org/10.1177/1741143216665838

Nguyen, H. and Van, B. (2018). University strategic research planning: a key to reforming university research in Vietnam? Studies in Higher Education, 43(12), 2130-2147. Doi: https://doi.org/10.1080/03075079.2017.1313218

Organización de las Naciones Unidas para la Educación, la Ciencia y la Cultura [Unesco] (2019). ¿Cuánto invierte su país en investigación y desarrollo? Instituto de Estadística de la UNESCO. Recuperado de http://uis.unesco.org/apps/visualisations/researchand-development-spending/\#!lang=es 


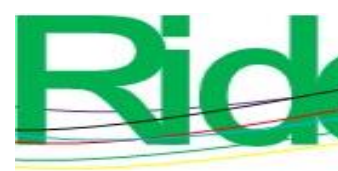

Revista Iberoamericana para la
Investigación y el Desarrollo Educativo
ISSN $2007-7467$

Ortiz, J., Topete, C. y Bustos, E. (2018). Efecto de las políticas educativas en la formación de investigadores en los programas federales de doctorado en ciencias administrativas en México. RIDE Revista Iberoamericana para la Investigación y el Desarrollo Educativo, 9(17), 462-493. Doi: https://doi.org/10.23913/ride.v9i17.389

Pantoja, M. (2019). Indicadores de desempeño académico como predictores de captación de recursos financieros. Convergencia Revista de Ciencias Sociales, 79(1), 1-27. Doi: https://doi.org/10.29101/crcs.v0i79.9222

Peinado, J., Cerecedo, M. y Jaramillo, D. (2015). Propuesta de un modelo de gestión del Capital Intelectual para los Centros de Investigación del IPN. Punto de Vista, 6(10), 135-157. Doi: http://dx.doi.org/10.15765/pdv.v6i10.768

Peinado, J., Cerecedo, M. y Jaramillo, D. (2016). El balanced scorecard, una herramienta de planeación estratégica en el Centro de Investigación e Innovación Tecnológica del Instituto Politécnico Nacional. UPIICSA Investigación Interdisciplinaria, 2(1), 3250. Recuperado de http://www.ruii.ipn.mx/index.php/RUII/article/view/19

Peinado, J. y Jaramillo, D. (2018). La eficiencia terminal del Centro de Investigación e Innovación Tecnológica. Revista Electrónica de Investigación Educativa, 20(3), 126134. Doi: https://doi.org/10.24320/redie.2018.20.3.1797

Peinado, J., Mayagoitia, V. y Cruz, C. (2019). Los grupos de investigación y su impacto en los factores que determinan la eficiencia terminal. Revista Dilemas Contemporáneos: Educación, Política y $\quad$ Valores, $7(1), \quad$ 1-26. $\quad$ Doi: https://doi.org/10.46377/dilemas.v28i1.1712

Peinado, J., Montoy, L. y Torres, Z. (2020). Estrategia de gestión para la generación de recursos en un centro de investigación y posgrado. Estudio de caso del CIITEC en el contexto de México. Acta Universitaria, 30(e2445), 1-27. Doi: https://doi.org/10.15174/au.2020.2445

Peinado, J. (2020). Experiencias del profesorado acerca del aprendizaje autónomo en estudiantes de modalidad a distancia y el uso de recursos digitales. RIDE Revista Iberoamericana para la Investigación y el Desarrollo Educativo, 10(20), 1-17. Doi: https://doi.org/10.23913/ride.v10i20.645

Román, J. (25 de junio de 2019). México rezagado en recursos para formar investigadores. La Jornada. Recuperado de https://www.jornada.com.mx/2019/06/25/sociedad/032n2soc 


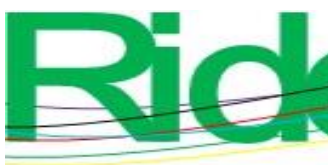

Revista Iberoamericana para la
Investigación y el Desarrollo Educativo
ISSN $2007-7467$

Rosas-Baños, M. (2019). Percepción de profesores investigadores sobre políticas educativas neoliberales en México. Revista Electrónica en Educación y Pedagogía, 3(5), 39-61. Doi: http://dx.doi.org/10.15658/rev.electron.educ.pedagog19.09030503

Rüschenpöhler, L. and Markic, S. (2019). Self-concept research in science and technology education-theoretical foundation, measurement instruments, and main findings. Studies in Science Education, 55(1), 37-68. Doi: https://doi.org/10.1080/03057267.2019.1645533

Taysum, A. (2016). Educational leaders' doctoral research that informed strategies to steer their organizations towards cultural alignment. Educational Management Administration \& Leadership, 44(2), 281-300. Doi: https://doi.org/10.1177/1741143213496660

Warshaw, J. and Upton, S. (2019). Hybrid logics in the resource strategies of US public research universities. Journal of Further and Higher Education, 1(1), 1-15. Doi: https://doi.org/10.1080/0309877X.2019.1680820

Weinberg, A., Trott, C. andy Sample, L. (2018). Who produces knowledge? Transforming undergraduate students' views of science through participatory action research. Science Education, 102(6), 1155-1175. Doi: https://doi.org/10.1002/sce.21453

Woiwode, H. and Froese, A. (2020). Two hearts beating in a research centers' chest: how scholars in interdisciplinary research settings cope with monodisciplinary deep structures. Studies in Higher Education, 45(7), 1-15. Doi: https://doi.org/10.1080/03075079.2020.1716321 